\title{
Data from: Examining the origins of heat content variability in the eastern North Atlantic subpolar gyre
}

\begin{tabular}{|c|c|}
\hline Variable name & Description \\
\hline Fig1_OHC_EN4 & $\begin{array}{l}\text { EN4 ocean heat content }\left(* 10^{21} \mathrm{~J}\right) \text { at monthly resolution from } \\
\text { January 1990-Dec } 2015\end{array}$ \\
\hline Fig1_OHC_ECCO & $\begin{array}{l}\text { ECCO version } 4 \text { release } 3 \text { ocean heat content }\left(* 10^{21} \mathrm{~J}\right) \text { at monthly } \\
\text { resolution from January 1992-Dec } 2015\end{array}$ \\
\hline Fig1_OHC_FLAME & $\begin{array}{l}\text { FLAME ocean heat content }\left(* 10^{21} \mathrm{~J}\right) \text { at monthly resolution from } \\
\text { January 1990-Dec } 2004\end{array}$ \\
\hline Fig2_latu & Latitude index to recreate Fig 2 (both $x$ and $y$ axes are latitude) \\
\hline Fig2_rlat & $\begin{array}{l}\text { Correlation coefficient between meridional heat transport } \\
\text { (calculated at trans-basin, zonal lines) in FLAME at different } \\
\text { latitudes. Dimensions are lat*lat. }\end{array}$ \\
\hline Fig3_Soutempflux & $\begin{array}{l}\text { Eulerian temperature flux (PW) across the southern face at } 30 \\
\text { day resolution, starting in January } 1995 .\end{array}$ \\
\hline Fig3_SPGtempflux & $\begin{array}{l}\text { Lagrangian temperature flux (PW) due to SPG-origin trajectories } \\
\text { across the southern face at } 30 \text { day resolution (January 1995- } \\
\text { December 2004). }\end{array}$ \\
\hline Fig3_STGtempflux & $\begin{array}{l}\text { Lagrangian temperature flux (PW) due to STG-origin trajectories } \\
\text { across the southern face at } 30 \text { day resolution (January 1995- } \\
\text { December 2004). }\end{array}$ \\
\hline Fig3_STGvolflux & $\begin{array}{l}\text { Lagrangian volume flux (Sv) due to STG-origin trajectories across } \\
\text { the southern face at } 30 \text { day resolution (January 1995-December } \\
\text { 2004). }\end{array}$ \\
\hline
\end{tabular}

